Rev. salud pública. 8 (1): 1-13, 2006

ARTÍCULOSIINVESTIGACIÓN

\title{
Análisis de la Política de Nutrición en Colombia
}

\author{
Rocío Ortiz-Moncada', María T. Ruiz-Cantero ${ }^{\mathrm{II}}$ y Carlos Álvarez-Dardet ${ }^{\mathrm{III}}$ \\ I Nutricionista Dietista, Ph. D. Salud Pública., M. Sc. Biología, M. Sc. Internacional en Medicina \\ Humanitaria. Área de Medicina Preventiva y Salud Pública-Universidad de Alicante. E-mail: \\ rocio.ortiz@ua.es \\ II Médica Cirujana, Ph. D. Medicina y Cirugía, M. Sc. Salud Pública. Área de Medicina Preventiva y \\ Salud Pública -Universidad de Alicante. E-mail: cantero@ua.es \\ ${ }^{\text {III }}$ Médico Cirujano, Ph. D. Medicina y Cirugía. Área de Medicina Preventiva y Salud Pública-Universidad \\ de Alicante. E-mail: carlos.alvarez@ua.es
}

Recibido 16 Septiembre 2005/Enviado para Modificación 12 Noviembre 2005/Aceptado 27 Enero 2006

\section{RESUMEN}

Objetivo Valorar la política de nutrición formulada en el Plan Nacional de Alimentación y Nutrición (PNAN) colombiano, 1996-2005, a partir de informantes clave (IC), planificadores y técnicos.

Materiales y Métodos Estudio descriptivo mediante encuesta transversal estructurada a 77 IC: 17 planificadores y 60 técnicos del PNAN. Variables: factores determinantes del problema alimentario, existencia de una política nutricional, valoración de políticas involucradas con seguridad alimentaria y, variables organizativas de la política. Se construyó un Índice de Posición (IP) que cuantificó las opiniones aportadas por los IC (0-0,33=valoración positiva; 0,34-0,67parcialmente/reajustarse; 0,68-1valoración negativa).

Resultados El $79 \%$ de informantes clave coinciden en que existe una Política de Nutrición, pero debe reajustarse (IP=0,50 planificadores, IP=0,54 técnicos). Falta acuerdo entre IC sobre la coordinación institucional, mientras que los planificadores opinan que hay coordinación entre un grupo reducido de entidades incluyendo la suya (IP=0,33); los técnicos opinan que no hay coordinación entre todas las instituciones $(\mathrm{IP}=0,75)$, además opinan que la estrategia de investigación no ha tenido éxito $(\mathrm{IP}=0,73)$.

Conclusiones Diez años después de la Política de Nutrición en Colombia, los IC opinan que debe reajustarse. Estrategias como la coordinación e investigación pueden optimizarse para alcanzar sus objetivos.

Palabras Clave: Política nutricional, formulación de políticas, personal administrativo (fuente: DeCS, BIREME).

\section{ABSTRACT}




\section{Nutritional policy and its framework in Colombia}

Objective Assessing the nutritional policy formulated in the 1996-2005 Colombian Food and Nutrition National Plan (FNNP) using key informants (KI), policy-makers and civil servants.

Material and Methods A descriptive cross-sectional structured survey of 77 $\mathrm{KI}$ : 17 policy-makers and 60 civil-servants from PNAN. Variables studied: determinant food factors, the existence of a nutritional policy, assessing policies involved with food security and organisational variables implicit in the policies. A Position Index $(\mathrm{PI})$ was constructed for quantifying $\mathrm{KI}$ assessment $(0-0.33=$ positive evaluation, $0.34-0.67=$ partial $/$ readjusted, $1=0.68-1=$ nega tive evaluation).

Results $79 \%$ of $\mathrm{KI}$ coincided in stating that there was a Nutritional Policy but that it should be readjusted (IP=0.50 policy-makers, $I P=0.54$ civil servants). $\mathrm{KI}$ did not agree about institutional coordination whilst policy-makers said that there was coordination between a reduced group of institutions, including themselves (IP=0.33); civil servants said that there was no coordination between any of the institutions $(\mathrm{IP}=0.75)$. They also said that the research strategy had been unsuccessful (IP=0.73).

Conclusions Ten years after the Nutritional Policy was introduced into Colombia KI say that it should be readjusted. Strategies such as coordination and research could be optimised so that their objectives can be reached.

Key Words: Nutrition policy, policy-making, administrative personnel (source: $\mathrm{MeSH}, \mathrm{NLM}$ ).

$\mathrm{E}$ n Colombia persiste la desnutrición en los niños menores de 5 años $(1,2)$. Está relacionada con problemas en la disponibilidad y acceso a los alimentos. Ya que mas del 50 \% de la población presenta bajos ingresos, y de estos el $20 \%$ no alcanza a cubrir las necesidades básicas de energía y nutrientes, incidiendo en la dificultad de conseguir la seguridad alimentaria de los hogares vulnerables más pobres (3). La FAO clasifica los países en desarrollo, de acuerdo al grado de seguridad alimentaria, en seis categorías, siendo la clase 1 los países en inseguridad alimentaria (4). Colombia se ubica en clase 5 de acuerdo al porcentaje de desnutridos y de disponibilidad per cápita de alimentos.

Estos problemas justificaron que el gobierno colombiano, siguiendo a su vez las directrices dadas en la Conferencia Internacional de Nutrición-CIN, de 1992, reorientara los programas nutricionales (5). Así, diseñó el Plan Nacional de Alimentación y Nutrición (PNAN), con dos estrategias de seguridad alimentaria, una dirigida a hogares rurales pobres, para mejorar sus 
condiciones de acceso a los alimentos, y otra, a grupos vulnerables para proteger y mejorar el estado nutricional (6).

Así como el caso colombiano, la desnutrición y la inseguridad alimentaria siguen afectando principalmente a los países en desarrollo, a pesar de los esfuerzos internacionales descritos en el diseño y puesta en marcha de políticas de nutrición en los últimos 40 años (7). Algunos autores atribuyen el escaso éxito alcanzado en los programas entre otros, tanto a la falta de planificación, evaluación y de coordinación entre los diferentes Ministerios a la hora de implementar las políticas de nutrición, como a la inequidad en la distribución de recursos -bienes y servicios-, y al desajuste económico y social que han sufrido estos países en la aplicación de los programas de estabilización económica y de ajuste (8-13).

Milio (14) considera importante tener en cuenta las condiciones organizativas para valorar las políticas, porque afectan a la respuesta que tendrán los ciudadanos a esa política. Específicamente se relaciona a: 1. Si realmente existe una política de nutrición, basada en un documento legislativo o técnico que la determinan. 2. La existencia de instituciones encargadas de planificar, poner en marcha y evaluar la política que permita conocer la factibilidad de mantener la política en el futuro, como las estrategias para llevar a cabo los objetivos de la política. 3. La participación intersectorial en el desarrollo de la política.

Por tanto, nuestro propósito a través de este estudio fue valorar la política de nutrición formulada en el Plan Nacional de Alimentación y Nutrición (PNAN) colombiano, a partir de la opinión de sus actores principales -los planificadores y técnicos- del PNAN.

\section{MATERIALES Y MÉTODOS}

Estudio descriptivo, a través de una encuesta transversal estructurada a informantes clave. La identificación de 77 informantes claves, se efectuó a partir de un estudio piloto realizado previamente (15). Los informantes se agruparon en dos niveles: 17 representantes del Grupo de Planificadores, diseñadores de la política. El Grupo de Técnicos, correspondiente a 60 informadores, profesionales de instituciones públicas y privadas del sector agrícola y de asistencia social, que tienen a cargo la ejecución y vigilancia, en el sector educativo. Además de asociaciones de comunidades científicas y profesionales de nutrición (15). 
El número de informadores claves seleccionados se obtuvo a través del criterio de saturación, que refleja la calidad y suficiencia de los datos. Este método garantiza que la muestra de informadores clave aporta la información sobre la que más hay acuerdo, y que nuevas incorporaciones a la muestra no aportarían datos nuevos (16).

Las variables recogidas se centran en la valoración de los aspectos organizativos y ambientales del PNAN, en que se formuló la política de nutrición. La construcción de las preguntas se basó en la definición de los análisis de políticas públicas, aplicadas al caso colombiano a partir del documento técnico del PNAN, sobre las estrategias, objetivos e instituciones involucradas en el PNAN $(17,18)$.

Se utilizó un cuestionario estructurado, tipo Likert autoadministrado. A los informadores clave, se les entrevistó individualmente, mediante cita con una carta explicativa del propósito del estudio y la metodología. También se entrevistaron profesionales de la nutrición en un encuentro científico organizado, previo acuerdo con los coordinadores del evento, que cedieron un espacio para la actividad.

Las variables estudiadas se agruparon en:

Factores determinantes del problema alimentario en Colombia: Inestabilidad económica, inestabilidad en el suministro de alimentos, problemas económicos limitantes de la adquisición de alimentos, privatización en la comercialización de insumos, bajo nivel de producción, condiciones ambientales y violencia

Existencia de una política de nutrición en Colombia: Valoración de las políticas involucradas con la seguridad alimentaria: alimentación y nutrición, agraria, salud, educación, comunicación, medioambiente, económica, social, y comercio exterior

Variables organizativas de la política: Existencia de instituciones responsables de llevar a cabo la planeación, puesta en marcha, evaluación y vigilancia del PNAN.

1. Cumplimiento de las estrategias propuestas en el PNAN: Coordinación, participación ciudadana, equidad, educación e investigación.

2. Coordinación institucional entre todas las instituciones, la mayoría de entidades incluyendo la del informador clave, la mayoría de entidades excluyendo la del informador clave, un grupo reducido de entidades in- 
cluyendo la del informador clave, un grupo reducido de entidades excluyendo la del informador clave.

3. El PNAN ha facilitado a las instituciones: Funciones bien definidas, competencias bien enmarcadas, compromisos claramente definidos.

Para el análisis de la información, se construyó un Índice de Posición (IP) que cuantifica la posición global de todas las opiniones aportadas por los informadores clave entre cero (valoración positiva) y uno (valoración negativa). El Índice de Posición permite ubicar en que lugar un conjunto de informadores clave califica el ítem investigado utilizando una escala simétrica (19).

El índice de posición (IP), se construyó de la siguiente forma: I=M-1/ k-1

$\mathrm{k}$

$\mathrm{M}=1 / n \sum \mathrm{i} n_{\mathrm{i}}$

$\mathrm{i}=1$

Donde $\mathrm{n}$ es el número de informadores clave entrevistados que valoraron

el ítem específico, utilizando una escala ordinal compuesta por las diferentes categorías. Se tiene que para cada ítem, el acumulado de puntos por los $\mathrm{n}$ informadores clave es la sumatoria del puntaje multiplicado por los n informadores que respondieron esa categoría. $\mathrm{K}$ es el total de categorías por ejemplo: sí, parcialmente, no; $\mathrm{y}, \mathrm{M}=1 / \mathrm{n}(1 \mathrm{n} 1+2 \mathrm{n} 2+3 \mathrm{n} 3)$. El promedio de puntos $\mathrm{M}$, por informador clave se mueve entre uno y tres. El valor mínimo se alcanza solo cuando todos los informadores clave caen en la categoría "sí", y el máximo cuando todos están en la última categoría "no".

\section{RESULTADOS}

Los planificadores como técnicos, coinciden que entre las causas principales del problema alimentario en Colombia están: la existencia de problemas económicos que limitan la adquisición de alimentos, la inestabilidad en la economía del país, la inestabilidad en el suministro de alimentos y finalmente las condiciones de violencia del país que no permiten soluciones al problema. Llama la atención que mientras que los planificadores opinan que el problema no se debe al bajo nivel de producción, los técnicos opinan que parcialmente (Tabla 1).

El $79 \%$ de los informadores coinciden en que existe una Política de Nutrición en Colombia. Hay divergencias en las respuestas entre los informadores clave, al momento de definir si las diferentes políticas que giran alrededor de la nutrición, deberían de permanecer, o si requerían de reajustarse, 
ó reformularse. Ya que los planificadores opinaron que todas demandaban ser reajustadas. Mientras que los técnicos opinaron que la política agraria, económica, social, y la de comercio exterior deberían de reformularse. Los dos grupos de informadores coinciden en que la política de alimentación y nutrición, objeto del presente estudio, debe solo reajustarse (Tabla 2).

Tabla 1. Factores que determinan el problema alimentario en Colombia, según los Informantes Clave (Índice Posición-IP)

\begin{tabular}{lcc}
\hline \multicolumn{1}{c}{ Factores } & Planificadores $n=17$ & $\begin{array}{c}\text { Técnicos } \\
n=60\end{array}$ \\
\hline Problemas económicos, limitan adquirir alimentos & 0,06 & 0,06 \\
\hline Inestabilidad en el suministro de alimentos & 0,28 & 0,30 \\
Inestabilidad económica & 0,16 & 0,17 \\
Condiciones de violencia & 0,31 & 0,22 \\
Condiciones ambientales, clima social no favorable & 0,33 & $\mathbf{0 , 4 5}$ \\
Privatización en la comercialización de insumos & $\mathbf{0 , 5 4}$ & $\mathbf{0 , 3 5}$ \\
Bajo nivel de producción & 0,71 & $\mathbf{0 , 4 9}$ \\
\hline Si IP=0,00-0,33; Parcialmente IP=0,34-0,67; No IP=0,68-1,00 &
\end{tabular}

Tabla 2. Valoración de las políticas de seguridad alimentaria, según Informantes Clave (Índice Posición-IP)

\begin{tabular}{lcc}
\multicolumn{1}{c}{ Políticas } & $\begin{array}{c}\text { Planificadores } \\
\mathrm{n}=17\end{array}$ & $\begin{array}{c}\text { Técnico } \\
\mathrm{s} \\
\mathrm{n}=60\end{array}$ \\
\hline Alimentación y Nutrición & 0,50 & 0,54 \\
Salud & 0,53 & 0,53 \\
Educativa & 0,61 & 0,66 \\
Comunicaciones & 0,61 & 0,58 \\
Medio Ambiente & 0,54 & 0,65 \\
Agraria & 0,67 & $\mathbf{0 , 7 4}$ \\
Económica & 0,67 & $\mathbf{0 , 7 3}$ \\
Social & 0,64 & $\mathbf{0 , 6 9}$ \\
Comercio Exterior & 0,60 & $\mathbf{0 , 7 2}$ \\
\hline Reajustarse IP=0,34-0,67; Reformularse $\mathrm{IP}=\mathbf{0 , 6 8 - 1 , 0 0}$
\end{tabular}

Los informantes clave, en general, están de acuerdo que en Colombia, existe una institución encargada de cuidar la política, tanto en su planeación, como en la puesta en marcha. Difieren sólo en el aspecto de la evaluación y vigilancia, ya que mientras los técnicos opinan que si existe una institución responsable de evaluar y vigilar, los planificadores opinan que parcialmente (Tabla 3).

En relación con el cumplimiento de las estrategias propuestas en el plan para lograr los objetivos del PNAN, los planificadores y técnicos coinciden 
en que las estrategias de coordinación, participación ciudadana, equidad y educación planteadas en el PNAN, han permitido parcialmente el acceso a los alimentos. En relación con la estrategia de la investigación los planificadores opinan que parcialmente, mientras que los técnicos definitivamente opinan que esa estrategia no ha contribuido en el acceso a los alimentos (Tabla 3).

Tabla 3. Valoración sobre aspectos organizativos de la política de nutrición en Colombia. Índice Posición-IP

\begin{tabular}{lcc}
\hline \multicolumn{1}{c}{ Valoración de la política de nutrición-PNAN } & $\begin{array}{c}\text { Planificadores } \\
\mathrm{n}=17\end{array}$ & $\begin{array}{c}\text { Técnico } \\
\mathrm{S}\end{array}$ \\
$\mathrm{N}=60$
\end{tabular}

Por otra parte, en relación con la coordinación intersectorial, el PNAN en su planteamiento propone que para el cumplimiento de los objetivos de la política, se requiere que las instituciones entre los diferentes sectores, logren entre ellas un proceso de acuerdos que permita la consecución de sus responsabilidades delimitadas a sus funciones. Los resultados muestran diferencias marcadas entre los planificadores y técnicos. Los planificadores opinaron que lograron este proceso de acuerdos entre un grupo reducido de entidades, incluyendo la de ellos mismos. Mientras que los técnicos opinan que no se dio 
una coordinación entre todas las instituciones involucradas en el PNAN. Los informadores opinan que sus instituciones han logrado parcialmente funciones bien definidas, competencias bien enmarcadas y compromisos claramente definidos (Tabla 3).

\section{DISCUSIÓN}

A pesar de la existencia de una política de nutrición en Colombia, persisten los principales factores determinantes del problema alimentario, tal como el relacionado con el suministro de alimentos y el relacionado con problemas económicos que limitan su adquisición. Según los informantes clave la política debe reajustarse. En este sentido, plantean problemas de falta de investigación y de instituciones responsables de la vigilancia y evaluación del PNAN, problemas de coordinación y de acuerdos interinstitucionales para lograr los objetivos de seguridad alimentaria nacional. Ligado a esto, a una falta de definición de las funciones, de los compromisos de cada institución y de sus competencias. Además, la opinión de los informantes clave es que el resto de políticas involucradas en la seguridad alimentaria deben reajustarse o incluso reformularse, como la económica, la social y la de comercio exterior.

En el estudio se presentaron limitaciones en el proceso de selección de expertos, especialmente en el grupo de planificadores. Algunas instituciones planificadas como participantes en el PNAN, para la ejecución de los programas de seguridad alimentaria en hogares rurales desaparecieron en las labores de reestructuración del Estado o, en su efecto hubo supresión de cargos con responsabilidades en el PNAN. Por tanto, el grupo de planificadores entrevistados se centró básicamente en los seleccionados en el estudio piloto. El grupo de ejecutores se limitó a instituciones públicas y privadas de áreas geográficas del centro y costa del país. Buscando la mayor representación posible de los actores principales del PNAN, se planearon entrevistas en congresos, seminarios y reuniones previamente organizados, donde participaron informadores de todos los lugares del país. Además, se entrevistaron expertos hasta la saturación de las respuestas, lo que garantiza que las opiniones más comunes y frecuentes han sido recogidas.

Las metas del milenio (MM) plantean disminuir a la mitad el porcentaje de personas que sufren desnutrición global entre 1990 y 2015 (20). En Colombia ha disminuido del 8,4 \% en 1995 al 6,7 \% en el año 2000, habiendo cumplido con el objetivo propuesto en el PNAN para el ámbito urbano, pero no para el rural, pues se mantiene en 8,9 \% (2,21). Por otra parte, no se ha cumplido con la MM para la desnutrición crónica, pues se cifra en un $13,5 \%(21)$. 
Por lo tanto, a pesar de que los informantes clave están de acuerdo en que en Colombia existe una política de nutrición, persisten según ellos condiciones ambientales relacionadas con factores externos e internos a la política, que determinan el problema alimentario.

Entre los factores externos que inciden en la política interna de nutrición se reitera el económico. En concreto, el hecho de que la economía del país no sea estable influye en la inestabilidad en el suministro de alimentos y limita su adquisición. Se han evidenciado resultados similares al colombiano (2225), donde factores externos como la liberalización del mercado de productos agrícolas ha tenido impacto negativo sobre la economía interna de países en desarrollo relacionada con la producción de alimentos y productos agropecuarios. Estos países han tenido que competir con los industrializados sin que su sector agrícola estuviera adecuadamente preparado. En consecuencia, la producción ha disminuido, con el consiguiente deterioro de la seguridad alimentaria entre los grupos vulnerables. Por otro lado, los informadores clave coinciden en que las condiciones de violencia en Colombia son el principal factor interno que dificulta la solución del problema alimentario.

Los resultados sobre los factores externos y los de violencia como factor interno son consistentes con las principales barreras identificadas en un estudio Delfos realizado junto con la presente investigación (15). Los análisis publicados resaltan que, los factores económicos posiblemente incidieron sobre las condiciones sociales en el campo y sobre el desplazamiento de la mano de obra calificada de los campesinos hacia zonas de cultivos ilícitos, centros urbanos y a su incorporación en grupos organizados no reconocidos por el gobierno nacional. Esto ha generado desplazamientos forzados de la población más pobre y vulnerable hacia las ciudades e inseguridad ciudadana y alimentaria (26). Quizás por ello, los planificadores y técnicos coinciden en que la política de alimentación y nutrición deben reajustarse. Y más aún, los técnicos enfatizan que las políticas agraria, económica, social y de comercio exterior incluso deberían de reformularse.

Para diseñar y ajustar la política, Milio específica que ante las presiones del mercado internacional y los cambios en la visión del mundo agrícola, se debe tener en cuenta las consecuencias de las negociaciones ante las fuerzas competitivas. Como también, para participar plenamente en las negociaciones, es necesario evaluar las propuestas avanzadas por otros países, y plantear sus propias posiciones de negociación de acuerdo con las capacidades internas agrarias (14). En consecuencia, es importante resaltar el contexto histórico político y económico del que parten las políticas nutrición. Así, la política de nutrición en Noruega y su institucionalización se formuló dentro del 
contexto de la política agraria (27). Quizás por ello, después de 10 años, a pesar de los conflictos internos de la propia política que dificultaron su puesta en marcha y mantenimiento, continúa el marco conceptual de sus objetivos productivos, y los resultados preliminares muestran cumplimiento de los mismos (14). En el caso de Chile la política de nutrición se formuló en el marco de la política de salud (28). Mientras que en Colombia, el ámbito de formulación de la política de nutrición fue el de la política social. Los diferentes estudios revisados evidencian que el gobierno de Colombia, dentro de un marco de referencia constituido por la Conferencia Internacional de Nutrición (CIN) de 1992 y la Cumbre Mundial de Alimentos (CMA) de 1996, y la presión de la política social internacional para reducir la desnutrición y el hambre en los países en desarrollo, inició la formulación del PNAN, puesto en marcha en 1996, y que ha abarcado dos periodos de cambio de gobierno 1996-1998 y 1999-2005 (29). Este marco de desarrollo de la política de nutrición probablemente se asocie al mayor desarrollo de los programas asistenciales de complementación alimentaria dirigidos a grupos vulnerables que de los programas productivos dirigidos a zonas rurales.

Las MM plantean la necesidad de determinar los medios y métodos para lograr la consecución de sus objetivos nutricionales (20). A través de la valoración que emiten los informadores clave sobre los aspectos organizativos de la política de nutrición se pueden identificar aquellos que son susceptibles de optimizar, y elaborar nuevas estrategias para mejorar la seguridad alimentaria, entre ellas las relacionadas con la coordinación y la investigación.

Planificadores y técnicos opinan que existen instituciones responsables de llevar a cabo la planeación y puesta en marcha, y vigilancia y evaluación del PNAN. Pese a ello, con la excepción de informes técnicos (30), aún no se ha evaluado. A diferencia de la política de nutrición de Noruega que planificada en 1975, y desarrollada entre 1976 y 1984, comenzó su evaluación en los 80 hasta concluirla en 1990 (14).

Es posible que a pesar de la existencia de las instituciones, estas no tengan completamente definidas sus funciones, sus competencias y sus compromisos, como opinan los informantes clave. Probablemente esto está relacionado con el hecho de que el Comité Nacional de Seguridad alimentaria (CONSA), creado en 1995, para la coordinación y vigilancia del PNAN, y compuesto por representantes de las instituciones implicadas en el mismo, debería reajustar y desarrollar las tareas para las que fue creado. Estas quizás han sido dificultadas por situaciones complejas similares a las sufridas por la política de nutrición en Noruega, especialmente en las instituciones sobre las que recaía la supervivencia de la política (31). 
Nancy Milio explica que durante el desarrollo de la política de nutrición de Noruega, el Consejo Nacional de Nutrición (CNN)-organización planificadora y cuidadora de la Política Nacional de Nutrición durante décadas- tuvo que luchar contra las barreras creadas por los intereses parciales de los actores pertenecientes a las entidades comprometidas en la alimentación, hasta que consiguieron que la Política de Nutrición estuviera en la agenda de los gobernantes (14).

Además, los informadores clave opinan que la estrategia de coordinación del PNAN se ha cumplido solo parcialmente. A esta valoración se debe sumar el que planificadores y técnicos difieren en el cómo se llevó a cabo la coordinación sectorial. Pues mientras que los planificadores opinan que existe la coordinación entre un grupo reducido de entidades incluida la suya, los técnicos opinan que no hay coordinación entre todas las instituciones involucradas en el PNAN.

Los resultados del estudio muestran discrepancia entre los informadores clave en la valoración que hacen de la estrategia de investigación. Los técnicos opinan que ésta estrategia no fue exitosa mientras que los planificadores la valoran parcialmente eficaz en el logro de los objetivos del PNAN.

La importancia de la investigación en el análisis de políticas de nutrición se ha resaltado en la literatura internacional, tanto las que presentan una orientación individual, dirigidas hacia el cambio de comportamiento como las que se orientan hacia cambios más estructurales. Sin embargo, las evaluaciones de políticas de nutrición y alimentación son escasas, y se refieren básicamente a países desarrollados. Una de las razones es por falta de presupuestos adecuados para la evaluación (32,33), pero Klepp y Foster aducen (34) que las políticas propuestas no se basan en proyectos de investigación científica, sino que se van realizando cambios sobre la marcha de los programas, no existiendo un límite de la política -donde comienza y donde termina-, lo cual hace muy difícil evaluar el impacto de la misma •

Agradecimientos. La ayuda y estímulo de los informantes clave fueron las bases para la realización del trabajo. El estudio ha sido posible gracias a las Ayudas de la AECI Intercampus y del Vicerrectorado de Coordinación y Comunicación de la Universidad de Alicante para la consolidación de Convenios. Como también a la Ayuda del Proyecto de Observatorio de Políticas Públicas y Salud (OPPS), financiado por la Generalitat Valenciana para la divulgación de resultados.

\section{REFERENCIAS}


1. Mora JO, De Paredes B, De Navarro L, Rodríguez E. Consistent improvement in the nutritional status of Colombian children between 1965 and 1989. Bull Pan Am Health Organ 1992;26(1):1-13.

2. ENDS 1995. Encuesta Nacional de demografía y salud Colombia. Bogotá: Departamento Nacional de Planeación; 1995.

3. Departamento Nacional de Planeación DNP. Informe técnico - Colombia. Bogotá: Dirección Desarrollo Social-DNP; 1994.

4. FAO. Evaluación de situación de la seguridad alimentaria mundial. Informe científico del comité de Seguridad Alimentaria Mundial de la FAO. 1998 (Junio 2-5. $24^{\circ}$ periodo de sesiones. [Internet] Disponible en: http://www.fao.org/ docrep/meeting/W8499s.htm. Consultado Mayo 15 de 2000.

5. WHO/FAO. World declaration and plan of action for nutrition. FAO/WHO: International Conference on Nutrition, Roma. Roma: Food and Agriculture Organisation; December 1992.

6. Ministerio de Salud, Departamento Nacional de Planeación (DNP). Plan Nacional de Alimentación y Nutrición -PNAN 1996-2005. Bogotá: DNP, Documento Conpes 2847; 1996.

7. FAO. El estado de la inseguridad alimentaria en el mundo 2004. Informe 2004. [Internet] Disponible en: http://www.fao.org/waicent/search/simple_s_ result_1252. Consultado Abril 15 de 2005.

8. Bengoa JM. Evolución de las políticas y programas de nutrición en América Latina. An Ven Nut 1988;1:194-201.

9. Rivera JA, Habicht Jean-P. The recovery of Guatemalan children with mild to moderate wasting: factors enhancing the impact of supplementary feeding. Am J Public Health 1996;86(10):1430-1434.

10. Walker A. The persistence of poverty under welfare states and the prospects for abolition. Inter J Health Serv 1992;22(1):1-17.

11. Lobao LM, Thomas P. Political beliefs in an era of economic decline: farmers attitudes toward state economic intervention, trade, and food security. Rural Sociol 1992;57(4):453-475.

12. Kaluski DN, Ophir E, Amede T. Food security and nutrition: the Ethiopian case for action. Public Health Nutr. 2002 Jun;5(3):373-81.

13. Baris E, McLeog K. Globalization and international trade in the twenty-first century: opportunities for and threats to the health sector in the South. Inter J Health Serv 2000;30(1):187-210.

14. Milio N. Nutrition policy for food-rich countries: A strategic analysis. Baltimore: The Johns Hopkins University Press; 1990.

15. Ortiz MR, Álvarez-Dardet C, Ruíz MT, Gascón E. Identificación de barreras a las políticas de nutrición y alimentación en Colombia: estudio por el método Delfos. Rev Panam Salud Pública 2003;14(3):186-191.

16. Fernández MJ. Introducción a la Investigación Cualitativa. FMC-Formación Médica Continuada en Atención Primaria. Servei Catala de la Salut Sector S. Actualizaciones. Barcelona. 1995;2 (1):23-28.

17. Meny I, Thoenig JC. (Traducción Morata F.) Las Políticas públicas. Barcelona 
(versión española): Editorial Ariel Ciencia Política; 1992.

18. Garrett MJ. An introduction to national futures studies for policymakers in the health sector. World Health Statist. Quart 1994;47(3-4):101-117.

19. Silva Aycaguer LA. Escalas e Indicadores. En: Cultura estadística e investigación científica en el campo de la salud: una mirada crítica. Madrid: Editorial Díaz de Santos; 1998. pp. 43-57.

20. Reddy S, Heuty A. Achieving the millennium development goals: a review and strategy. United Nations Development Programmme. Draft 2.3; April $4^{\text {th }}$ 2004.

21. ENDS 2000. PROFAMILIA. Encuesta Nacional de Demografía y Salud Colombia. Bogotá: Profamilia; 2000.

22. Ford JRD. Guyana's food performance in a Caribbean context: lessons for food security policy. Food Policy 1992;17(5):326-336.

23. Pinckney TC. Is market liberalization compatible with food security? Storage, trade and price policies for maize in Souther Africa. Food Policy 1993;18(4):325-333.

24. Krissof B, Trapido P. Food and agricultural policy reform: the case of Venezuela. Food Policy 1991;16(2):140-151.

25. Omamo SW, Lynam JK. Agriculture science and technology policy in Africa. Research Policy 2003;32:1681-1694.

26. Gómez MA. Jalonando el futuro del sector rural: Informe al Congreso 19941998. Bogotá: Ministerio de Agricultura y Desarrollo Rural de Colombia; 1998.

27. Kjaernes U. Experiences with the Norwegian nutrition policy. Appetite 2003; 41:251-257.

28. Albala C, Vio F, Kain J, Uauy R. Nutrition transition in Chile: determinants and consequences. Public Health Nutr 2002; 5:123-128.

29. DNP. Bases del Plan Nacional de Desarrollo 1998-2002. Cambio para Construir la Paz. Bogotá: TM Editores; 1998.

30. Instituto Colombiano de Bienestar Familiar-ICBF. Plan Nacional de Alimentación y Nutrición 1996-2005: Informe de evaluación 1996-2002, resumen ejecutivo. Informe del Comité técnico CONSA -Comité Nacional de Seguridad Alimentaria-. Bogotá: ICBF: 2003: Septiembre. [Internet]. Disponible en: http://www.icbf.gov. Consultado Mayo 30 de 2004.

31. Mannan MA. On food and nutrition policy activities in the USA, Australia, and Norway. J Health Popul Nutr. 2004;22(2):191-202.

32. Pelletier DL, Shrimpton R. The role of information in the planning, management and evaluation of community nutrition programmes. Health Policy Plan 1994; 9(2):171-184.

33. Mannan MA. An evaluation of the national food and nutrition policy of Bangladesh. Food Nutr Bull. 2003; 24(2):183-92.

34. Klepp KI, Foster JL. The Norwegian nutrition and food policy: an integrated policy approach to a public health problem. J Public Health Policy. 1985; 6(4):447-463. 
